\title{
Clinical outcome of neonates with nosocomial suspected sepsis treated with cefazolin or vancomycin. A non-inferiority, randomized, controlled trial
}

\author{
José M. Ceriani Cernadas, M.D. ${ }^{a, b}$, Silvia Fernandez Jonusas, M.D. ${ }^{a}$, \\ Maritza Márquez, M.D. ${ }^{a}$, Armando Garsd, Ph.D. ${ }^{c}$ and Gonzalo Mariani, M.D. ${ }^{a}$ \\ Collaborators: Amorina Pardo, M.D., Adrián Aguilar, M.D., Andrea Lew, M.D. and \\ Marcelo Armadans, M.D.
}

\begin{abstract}
Background. Nosocomial infections are a major problem in Neonatal Intensive Care Units. Coagulase negative Staphylococcus (CONS) is the most common causative agent. We evaluated the efficacy of cefazolin versus vancomycin as initial therapy for neonates with presumptive clinical signs of nosocomial sepsis probably caused by CONS.

Methods. Hospitalized newborns infants with clinical signs of very probable bacterial sepsis were included. Two groups were randomly assigned according the initial antibiotic therapy: cefazolin group (CG) or vancomycin group (VG). The primary analysis was performed on an intention-to-treat basis. The main outcome measure was the clinical outcome of infants in both groups at the end of antibiotic treatment Results. We analyzed 109 newborns, 52 in CG and 57 in VG. Baseline characteristics were similar among groups. The percentage of neonates with adequate outcome was $92 \%$ in the CG and $86 \%$ in the VG: difference: $6 \%$ (95\% CI: $-7 \%$ to $19 \%$, $\mathrm{p}$-value non-inferiority, $\mathrm{p}=0.007)$. Seven infants died in the CG $(13.5 \%)$ and and $11(19.2 \%)$ in the VG; no significant difference ( $\mathrm{p}=0.45)$.

Conclusion. Cefazolin was not inferior to vancomycin in achieving an adequate clinical outcome in newborn infants with confirmed or highly probable nosocomial sepsis

Keywords:Nosocomial infections, newborns, cefazolin, vancomycin, coagulase negative, Staphylococcus.

ClinicalTrials.gov: NCT01867138.
\end{abstract}

http:/ /dx.doi.org/10.5546/aap.2014.eng.308

E-mail Address:

José M. Ceriani

Cernadas M.D.:

jose.ceriani@

hospitalitaliano.org.ar

Conflict of Interest:

None.

ClinicalTrials

Registration:

Neonatal suspected

sepsis treated

with cefazolin

or vancomycin,

NCT01867138.

Received: 1-28-2014

Accepted: 3-27-2014
(CONS) is the most common causative agent in hospital acquired or late onset neonatal sepsis in preterm infants with a birth weight of less than $1,500 \mathrm{~g}$. Studies by the National Institute of Child Health and Human Development Neonatal Research Network, USA, have shown that between $55 \%$ and $58 \%$ of hospital acquired bacteremias in very low birth weight preterm infants are caused by CONS. ${ }^{1,2}$ Another study collected data from 29 neonatal intensive care units, showing that CONS was the cause of nosocomial sepsis in $48.3 \%$ of newborn infants. ${ }^{3}$ A recent study showed a prevalence of $45 \%{ }^{4}$ with other studies showing rates as high as $70 \%{ }^{5}$

Vancomycin is one of the antibiotics of choice for presumed CONS sepsis and other Grampositive organisms, and is used in the initial empiric treatment for late onset or nosocomial sepsis, ${ }^{5-7}$ usually in association with a broad spectrum antibiotic. However, wide spread use of vancomycin may entail further untoward consequences, including adverse events, prolonged use of intravenous lines, and the development of bacterial resistance, a major public health concern..$^{8-9}$

One of the most probably causes of the increase in antimicrobial resistance is the extensive use of vancomycin in hospitals from the emergence of meticiline resistance in the late $70 \mathrm{~s} .{ }^{6}$ For this reason, one of the strategies recommended to reduce vancomycin use is to replace it with alternative antibiotics. ${ }^{8,9}$ 
In order to validate this strategy, we have evaluated the effectiveness of empiric treatment with cefazolin versus vancomycin in newborn infants with presumptive clinical signs of hospital acquired bacterial sepsis probably caused by CONS.

We hypothesized that newborn infants with the presumptive diagnosis of nosocomial sepsis who received cefazolin as empiric treatment would have a clinical outcome not inferior to that of those treated with vancomycin.

\section{Study Design}

This was a prospective, active-controlled, randomized, open-label, two-arm, parallel-group, non-inferiority study.

\section{PATIENTS AND METHODS}

The study was done in the Division of Neonatology at the Hospital Italiano de Buenos Aires, Argentina, between March 2006 and August 2010. It was sponsored by the Carlos A. Gianantonio Foundation, a nonprofit organization, without external funding from the pharmaceutical industry.

The Hospital Ethics Committee on Research Protocols approved the study (protocol number 563) and parents of all participating infants signed the informed consent.

Newborn infants hospitalized in the NICU who had clinical signs of highly probable nosocomial or late onset bacterial sepsis were eligible to be enrolled in the study.

Newborn infants included were older than three days and, according to attending physicians and to the guidelines of the NICU, had to have an indication for initial empiric treatment with vancomycin for suspected bacterial sepsis. At least one blood culture was required prior to receiving the antimicrobial treatment.

Newborn infants were excluded if, at the time of inclusion, they had been treated with vancomycin during the week before, or if they had been referred from other hospitals, and were already receiving antibiotic treatment.

Allocation of patients to treatment: newborn infants who met inclusion criteria and none of the exclusion criteria were randomly allocated to one of the following two treatment groups: cefazolin and amikacin (cefazolin group,[CG]), or vancomycin and amikacin (vancomycin group [VG]).

Antibiotics were administered intravenously at a dose and dosing interval depending on the gestational and postnatal ages. Current standard management and ancillary tests were performed on newborn infants with suspected bacterial sepsis.

The therapy duration was 7 to 10 days according to the clinical outcome and the presence of a second positive blood culture within 72 hours of starting treatment. If the clinical course was satisfactory with normalization of laboratory tests initially altered, antibiotics were suspended after seven days of therapy. In infants with a second positive blood culture or more torpid evolution according to the discretion of the attending physician, treatment continued until 10 days.

Primary outcome: clinical outcome of infants in both groups at the end of antibiotic treatment.

Secondary outcome: Percentage of infants initially assigned to cefazolin group that 72 hours later were switched to vancomycin.

\section{Definitions}

Confirmed sepsis was defined as a clinical condition compatible with bacterial infection with a positive blood and/or cerebrospinal fluid culture.

Highly probable sepsis was defined as a negative culture in a newborn infant with clinical signs of sepsis and two or more of the following diagnostic test results: $<5000$ white blood cells / $\mathrm{mm}^{3},<1500$ neutrophils $/ \mathrm{mm}^{3}$, immature to total neutrophil (I/T) ratio $\geq 0.2, \mathrm{C}$-reactive protein $>10 \mathrm{mg} / \mathrm{L}$, and platelet count $<100000 / \mathrm{mm}^{3}$.

Nosocomial or late onset sepsis was considered when clinical signs of bacterial infection were present after the third day of life and before hospital discharge.

\section{Clinical examination}

The clinical examination to assess the status of the main outcome variable was performed at the end of antibiotic treatment (between 7 to 10 days from the initiation of treatment). Each newborn infant was examined by two of the investigators independently to establish whether the clinical outcome was adequate or inadequate. In order to achieve greater objectivity, the clinical examination was performed using the following criteria:

Adequate: when the newborn infant had normal clinical parameters, a good general condition, appropriate tolerance to gastric feeding, normal temperature, negative blood and CSF cultures, and normalized results for the lab tests performed at the onset of the treatment. 
Inadequate: when one or more of the following were present: clinical course with persistence of any of the signs of sepsis, positive blood or CSF cultures after 72 hours from the onset of treatment, persistence of abnormal lab tests, and death by sepsis.

\section{Randomization}

The randomization and organization of the process were supervised by one member of the research team (JMCC) who was not directly involved in the clinical care of patients on study; therefore the randomization process was blinded. The list was generated via a computer software by assembling random blocks of variable size $(6,8$ and 10). Treatment group allocation details were stored safely, in sequentially numbered, opaque, sealed envelopes.

\section{Rescue of patients failing cefazolin treatment}

Patients with an inadequate 72 hours response to cefazolin were crossed over to receive vancomycin. The adjudication of response at 72 hours was delineated in a well-defined algorithm. To minimize bias, the final decision to switch treatments, initiated by the pediatrician in charge, required secondary review and agreement of another participating investigator as well as the attending NICU physician.

\section{Sample size}

For the purpose of our sample-size calculation, we assumed primary treatment adequate response rates of $90 \%$ in both treatment arms. With the use of the normal approximation to the binomial distribution, 112 cases in each treatment arm would be required to demonstrate that the upper limit of the $95 \%$ confidence interval (CI) for the treatment difference ([VG] - [CG]) in primary adequate response rates had to be smaller than $10 \%$ (power: $80 \% ; \alpha=0.05$ ). ${ }^{10,11}$ Given the size of the projected sample, it was decided to adaptively approach the study in two sequential steps with statistical adjustments to account for multiplicity of analyses. ${ }^{12}$ In the first stage, 115 neonates should be randomized and, if results confirmed or support the hypothesis, the study would end at this stage. If, however, tentative results were promising and future recruitment feasible, it could then be extended to a second (and final) stage based on a definitive sample size re-estimation. ${ }^{13,14}$

\section{Statistical analysis}

The main exposure was the antibiotic treatment in infants with nosocomial sepsis. The primary analysis was performed on an intention-to-treat basis. Several sensitivity analyses were also performed. The efficacy comparison between the VG and the CG was performed considering the differences in response rates between treatments and the $95 \% \mathrm{CI}$ for this difference relative to the non-inferiority tolerance $(10 \%)$ based on the fixed margin approach. ${ }^{15}$ Binomial outcome measures were tested using Wald test with continuity correction.

SAS software version 9.3 (SAS Institute Inc., Cary, NC, USA) was used for all analyses.

\section{RESULTS}

The total number of patients analyzed was 109. Of these, 52 were allocated to cefazolin and 57 received vancomycin (Figure 1). The two groups had homogenous baseline characteristics (Table 1) and similar clinical signs of sepsis (Table 2). The percentage of confirmed sepsis between the two groups was also similar: $38.4 \%$ $(20 / 52)$ in the CG and $38.6 \%$ in the VG $(22 / 57)$, (Table 3 ) as was the percentage of confirmed sepsis caused by CONS: 70\% (14/20) and 72.7\% (16/22) in the CG and VG groups, respectively. The difference in the distribution of cases of newborns with a second CONS positive blood culture at 72 hours was tree cases in the CG group (5.7\%) versus 5 cases in the VG group (9.4\%).

Other germs found in infants from both groups are shown in Table 3.

No adverse effects associated with the use of any of the antibiotics were detected, except in one infant in the vancomycin group who, after 7 days of treatment, showed a positive blood culture for Candida albicans and was medicated with amphotericin B.

The overall mortality rate was $13.5 \%(7 / 52)$ in the CG and $19.2 \%(11 / 57)$ in the VG $(\mathrm{p}=0.45)$. Mortality rate by sepsis was $4 \%$ and $9 \%$ in the cefazolin and vancomycin groups, respectively $(\mathrm{p}=0.44)$.

\section{Primary outcome variable}

The intention-to-treat analysis showed that clinical outcome was adequate in $92.3 \%(48 / 52)$ and $86 \%(49 / 57)$ of the patients in the CG and VG groups, respectively. The difference in percentages, $6.3 \%$ (95\% CI: -7 to $19.8 \%$ ), attained statistical significance for the non-inferiority test: $p=0.007)$. The per-protocol analysis confirmed the ITT data $(\mathrm{p}<0.001)$.

The number of cases with adequate clinical 


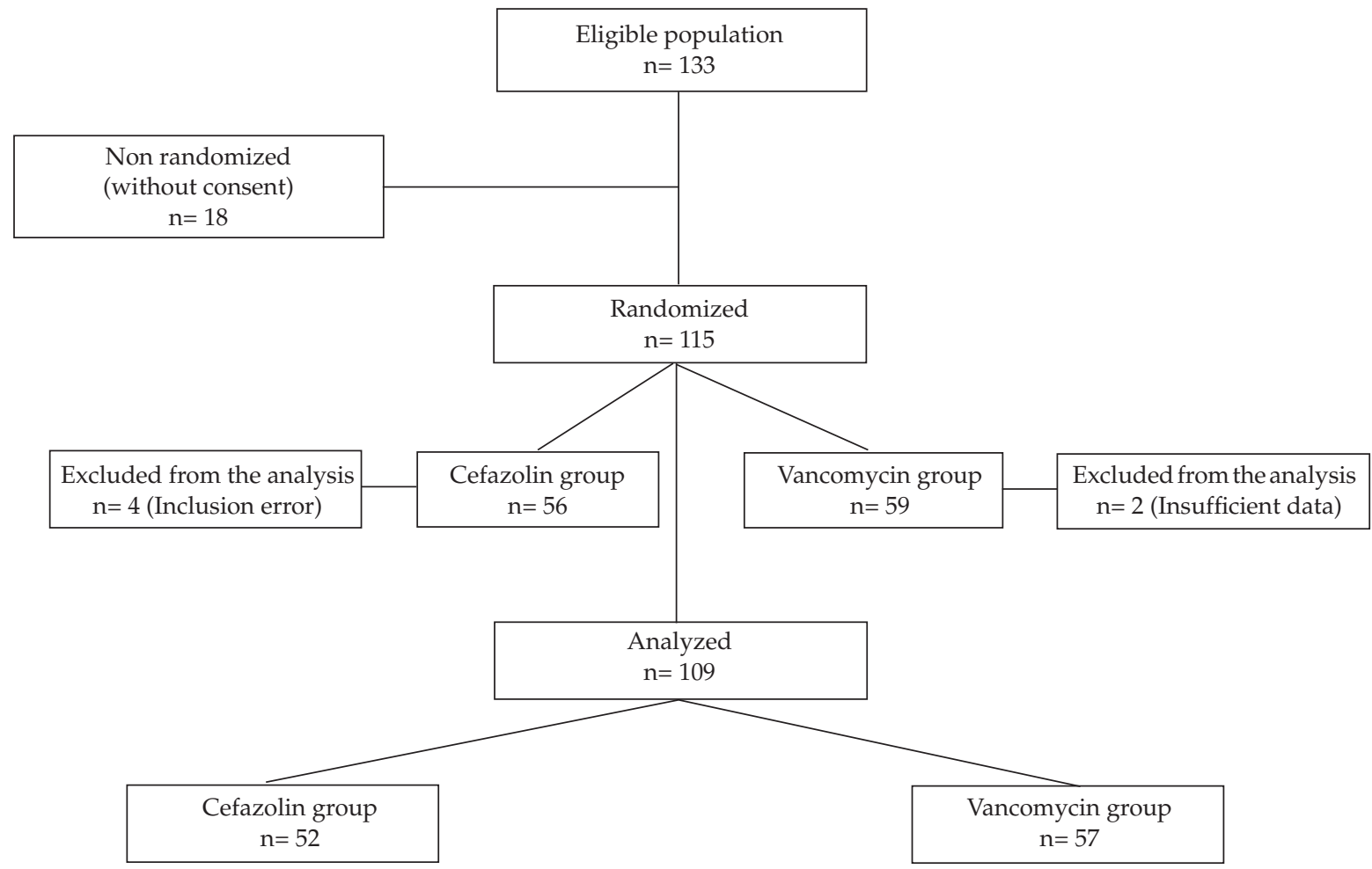

TABle 1. Population characteristics

\begin{tabular}{lccc}
\hline & Cefazoline group N= 52 & Vancomycin group N= 57 & $\mathbf{p}^{*}$ \\
\hline Maternal morbidity during pregnancy, n (\%) & $21(40.4)$ & $23(40.3)$ & 1.0 \\
Twin pregnancy, $\mathrm{n}(\%)$ & $4(7.7)$ & $4(7.0)$ & 1.0 \\
Referred newborns, $\mathrm{n}(\%)$ & $9(17.3)$ & $12(21.0)$ & 0.64 \\
Gestational age, weeks, mean (SD) & $31.2(5.1)$ & $31.5(4.7)$ & 0.83 \\
Prematurity (<37 weeks, n [\%]) & $42(80.7)$ & $47(82.4)$ & 1.0 \\
IUGR, $\mathrm{n}(\%)$ & $7(13.4)$ & $4(7.0)$ & 0.35 \\
Birth weight, g, mean (SD) & $1629.3(1001.5)$ & $1650.1(888.9)$ & 0.91 \\
Apgar at 5 minuts $<7, \mathrm{n}(\%)$ & $3(5.7)$ & $5(8.7)$ & 0.72 \\
\hline
\end{tabular}

IUGR: intrauterine growth restriction.

* Statistical significance of the respective difference, based on the exact chi squared test for the difference in binomial proportions and the two-sample Student's t test for the difference in gestational age and birth weight, respectively.

TABLE 2. Most common clinical signs of sepsis in both groups

\begin{tabular}{lccc}
\hline Clinical sign (N, \%) & Cefazoline group $\mathbf{N = 5 2}$ & Vancomycin group N= 57 & $\mathbf{P}^{*}$ \\
\hline Abdominal bloating and/or residue & $21(40.3)$ & $18(31.8)$ & 0.42 \\
Hyperthermia & $19(36.5)$ & $23(40.3)$ & 0.70 \\
Apnea & $17(32.6)$ & $18(31.5)$ & 1.0 \\
Shortness of breath & $17(32.6)$ & $24(42.1)$ & 0.33 \\
Tachycardia & $14(26.9)$ & $11(19.3)$ & 0.37 \\
\hline
\end{tabular}

* Statistical significance of the respective difference, based on the exact chi squared test for the difference in binomial proportions. 
outcome in CONS sepsis also showed similar values for both groups: $92.9 \%(13 / 14)$ in the CG and $87.5 \%(14 / 16)$ in the VG, although the sample size does not allow a definitive conclusion on the parity of the two treatments (95\% CI: $-22.4-33.1 \%$, $p$ value for the non-inferiority: $p=0.21$ ).

\section{Secondary outcome variable}

The percentage of newborn infants allocated to the CG who were switched to vancomycin at 72 hours was $17.3 \%$ (9/52 [95\% CI: 8.2 to $30.3 \%$ ]). The breakdown of these cases is as follows: two newborn infants were crossed over to VG because of an inadequate clinical outcome at 72 hours and two infants were switched to VG due to a second positive blood culture at 72 hours. The other five patients were rescued in response to the presence of infections caused by other germs.

\section{DISCUSSION}

The results of this study tend to support the hypothesis that cefazolin is not inferior to vancomycin as initial empiric treatment for newborn infants with presumed nosocomial or late onset sepsis.

Clinical outcome at 7-10 days after the initiation of treatment was adequate for most cases in both groups. However, it is noteworthy that, although the difference was not statistically significant, the mortality rate for sepsis was more than two times higher in the vancomycin group. Furthermore, the frequency of second CONS positive blood culture at 72 hours was also higher in the vancomycin group.

Vancomycin is one of the most common antibiotics of choice for suspected late onset neonatal sepsis, ${ }^{6,7}$ however, there is no sound evidence from randomized studies in favor of one particular antibiotic being optimal for this condition. Even several aspects of its pharmacology and safety are unknown in newborn infants, ${ }^{16}$ its use has markedly increased in the last 20 years..$^{6-9,16}$

The major problems with the excessive use of vancomycin is the emergence of Gram-positive resistance, a concern voiced by many authors in the last 20 years $8,9,16$.

The first publications about acquired bacterial resistance to glycopeptide antibiotics was reported in relation to CONS in the mid $80 \mathrm{~s} .{ }^{17}$ The emergence of vancomycin-resistant enterococci was published in $1988^{18}$ and this resistance quickly became a great concern. The biochemical and genetic mechanisms of resistance in enterococci were rapidly and comprehensively elucidated, unlike resistance mechanisms in the CONS, which still remain several uncertainties. Vancomycin-resistant enterococci became the main nosocomial pathogens, especially in the $\mathrm{USA}^{19}$, and most publications were focused on enterococci resistance, even in newborn infants, ${ }^{20}$ and on Staphylococcus aureus resistance, that also emerged in that years. ${ }^{21}$

In the following years, CONS resistance continued to be described ${ }^{16}$ and likewise, CONS strains with scarce susceptibility and partial sensitive to vancomycin were isolated; ;2-24 which might explain the persistent and inadequate clinical course and prolonged bacteremias in some newborn infants with CONS sepsis in spite of vancomycin treatment. Lower susceptibility has also been described in Staphylococcus aureus infections ${ }^{25}$.

Vancomycin-resistant CONS is more frequent in Staphylococcus haemolyticus and Staphylococcus

TABLE 3. Sepsis diagnosis

\begin{tabular}{|c|c|c|c|}
\hline $\begin{array}{l}\text { Degree of certainty } \\
\text { in sepsis diagnosis and } \\
\text { pathogens identified }(n, \%)\end{array}$ & $\begin{array}{l}\text { Cefazolin group } \\
\qquad \mathrm{N}=52\end{array}$ & $\begin{array}{l}\text { Vancomycin group } \\
\qquad N=57\end{array}$ & $\mathrm{p}$ \\
\hline $\begin{array}{l}\text { Confirmed } \\
\text { Blood culture }+ \\
\text { CSF }+\end{array}$ & $\begin{array}{c}20(38.5) \\
19 / 52(36.5) \\
2 / 45(4.4)^{*}\end{array}$ & $\begin{array}{c}22(38.6) \\
22 / 57(38.6) \\
1 / 48(2.1)^{* *}\end{array}$ & $\begin{array}{l}1.0 \\
0.85 \\
0.61\end{array}$ \\
\hline $\begin{array}{l}\text { CONS } \\
\text { Staphylococcus aureus } \\
\text { Gram negative bacilli }\end{array}$ & $\begin{array}{c}14(26.9) \\
3(5.8) \\
3(5.8)\end{array}$ & $\begin{array}{c}16(28.0) \\
2(3.5) \\
4(7)\end{array}$ & $\begin{array}{l}1.0 \\
0.66 \\
0.64\end{array}$ \\
\hline Very likely & $32(61.5)$ & $35(61.4)$ & 0.98 \\
\hline
\end{tabular}

CSF: cerebrospinal fluid.

CONS: coagulase-negative Staphylococcus.

* CSF was analyzed in 45 newborn infants, two had positive results, and one had positive blood and CSF cultures.

** CSF was analyzed in 48 newborn infants, one had positive blood and CSF cultures. 
epidermidis species, ${ }^{24}$ the latter one being one of the most common strains in late onset neonatal sepsis. In addition, some reports have pointed out that reduced vancomycin susceptibility would be more common in methicillin-resistant CONS. ${ }^{24}$

The risk of increased resistance to vancomycin have led to the implementation of several strategies, generally based on adopting a practice of judicious use of antibiotics, ${ }^{8}$ restricting their indications and using them for the shortest time possible. However, implementing such strategies in neonatal units is often difficult, especially because of the low specificity in the clinical diagnosis of sepsis, which results in antibiotics being very frequently indicated for "suspected sepsis."

Another recommended strategy is replacing vancomycin by other alternative antibiotics with a lower probability of resistance. ${ }^{5,6,9,26,27}$

In this study, we chose to evaluate replacement of vancomycin by a first-generation cephalosporin. It was selected based on prior studies that demonstrated that their effectiveness in neonatal sepsis caused by CONS and Staphylococcus aureus; furthermore, bacterial resistance to cephalosporins would be expected to be less frequent than with vancomycin. 5,6,24

Krediet et al. ${ }^{5}$ observed in a group of newborn infants with CONS sepsis, $90 \%$ of whom were methicillin-resistant mecA gene carriers, that cephalothin had the same effectiveness as vancomycin in terms of the clinical course of treated newborn infants. With the strategy of using cephalothin, they were able to reduce the use of vancomycin from $62 \%$ to $21 \%$ in two years.

Likewise, Brand $\mathrm{t}^{28}$ et al. observed that, in the induction of experimental endocarditis by CONS, the combination of cefazoline or cefpirone with rifampicin was as effective as the combination with vancomycin.

\section{CONCLUSIONS}

Although the sample size used limits the scope of drawing conclusions from our study, we were able to support the hypothesis that cefazolin is not inferior to vancomycin in terms of achieving an adequate clinical outcome in newborn infants with confirmed or highly probable nosocomial sepsis and, therefore, this antibiotic have the potential for replacing vancomycin at the stage of the initial empiric treatment. Because our study was exploratory, these results should be considered with caution until further studies confirm our findings.

\section{Acknowledgements}

The authors are grateful to Ian Scott and Charles Dowding for their critical review of an early manuscript. We also wish to thank the parents of the newborns enrolled in the study who trusted us.

\section{REFERENCES}

1. Stoll BJ, Hansen N, Fanaroff AA, Wright LL et al. Late onset sepsis in very low birth weight neonates: the experience of the NICHD Neonatal Research Network. Pediatrics 2002;110(2):285-91.

2. Fanaroff AA, Korones SB, Wright LL, Verter J et al. Incidence, presenting features, risk factors and significance of late onset septicemia in very low birth weight infants. Pediatr Infect Dis J 1998;17(7):593-8.

3. Sohn AH,Garrett DO,Sinkowitz-Cochran RL, Grohskopf LA et al. Prevalence of nosocomial infections in neonatal intensive care units patients: results from the first national point-prevalence survey. J Pediatr 2001;139(6):821-7.

4. Muller-Pebody B, Johnson AP, Heath PT, Gilbert RE et al. Empirical treatment of neonatal sepsis: are the current guidelines adequate? Arch Dis Child Fetal Neonatal Ed 2011;96(1):F4-8.

5. Krediet TG, Jones ME, Gerards LJ, Fleer A. Clinical outcome of cephalothin versus vancomycin therapy in the treatment of coagulase-negative staphylococcal septicemia in neonates: relation to methicillin resistance and mec A gene carriage of blood isolates. Pediatrics 1999;103(3):E29.

6. Venkatesh MP, Placencia F, Weisman LE. Coagulase-negative staphylococcal infections in the neonate and child: an update. Semin Pediatr Infect Dis 2006;17(3):120-7.

7. Gordon A, Jeffery HE. Antibiotic regimens for suspected late onset sepsis in newborn infants. Cochrane Database Syst Rev 5005;(3):CD004501.

8. Issacs D. Rationing antibiotic use in neonatal units. Arch Dis Child Fetal Neonatal Ed 2000;82(1):F1-2.

9. Chiu CH, Michelow IC, Cronin J, Ringer SA et al. Effectiveness of a guideline to reduce vancomycin use in the neonatal intensive care unit. Pediatr Infect Dis J 2011;30(4):273-8.

10. Blackwelder WC. "Proving the null hypothesis" in clinical trials. Control Clin Trials 1982;3(4):345-53.

11. Sachs L. Applied statistics: a handbook of techniques. 2nd ed. New York: Springer-Verlag;1984,p.333.

12. Blackwelder,WC,Chang MA.Samplesize graphs for "proving the null hypothesis". Control Clin Trials 1984;5(2):97105.

13. Denne JS. Estimation following extension of a study on the basis of conditional power. J Biopharm Stat 2000;10(2):13144.

14. Mehta CR, Pocock SJ. Adaptive increase in sample size when interim results are promising: A practical guide with examples. Stat Med 2011;30(28):3267-84.

15. Li G, Chi GYH. Inferiority index and margin in noninferiority trials. Stat Biopharm Res 2011;3(2):288-301.

16. Dehority W. Use of vancomycin in pediatrics. Pediatr Infect Dis J 2010;29(5):462-4.

17. Schwalbe RS, Stapleton JT, Gilligan PH. Emergence of vancomycin resistance in coagulase-negative staphylococci. $N$ Engl J Med 1987;316(15):927-31.

18. Uttley AH, Collins CH, Naidoo J, Giorge RC. Vancomycinresistant enterococci. Lancet 1988;1:57-8.

19. Centers for Disease Control and Prevention. Nosocomial enterococci resistant to vancomycin - United States, 19891993. MMWR 1997;42(30):597-9. 
20. Sherer CR, Sprague BM, Campos JM, Nambiar S et al. Characterizing vancomycin-resistant enterococci in neonatal intensive care. Emerg Infect Dis 2005;11(9):1470-2.

21. Smith TL, Pearson ML, Wilcox KR, Cruz Cet al. Emergence of vancomycin resistance in Staphylococcus aureus. Glycopeptide-Intermediate Staphylococcus aureus Working Group. N Engl J Med 1999;340(7):493-501.

22. Sieradzki K, Roberts RB, Haber SW, Tomasz A. The development of vancomycin resistance in a patient with methicillin-resistant Staphylococcus aureus infection. N Engl JMed 1999;340(7):517-23.

23. Center KJ, Reboli AC, Hubler R, Rotgers GL, Long SSl. Decrease vancomycin susceptibility of coagulase-negative staphylococci in a neonatal intensive care unit: evidence of spread of Staphylococcus warneri. JClin Microbiol 2003;41(10):4660-5.

24. Biavasco F, Vignaroli C,Varaldo PE. Glycopeptide resistance in coagulase-negative staphylococci. Eur J Microbiol Infect Dis 2000;19(6):403-17.
25. van Hal SJ, Lodice TP, Paterson DL. The clinical significance of vancomycin minimum inhibitory concentration in Staphylococcus aureus infections: a systematic review and meta-analysis. Clin Infect Dis 2012;54(6):755-71.

26. Karlowicz MG, Buescher ES, Surka AE. Fulminant lateonset sepsis in a neonatal intensive care unit, 1988-1997, and the impact of avoiding empiric vancomycin therapy. Pediatrics 2000;106(6):1387-90.

27. Lawrence SL, Roth V, Slinger R, Toye B et al. Cloxacillin versus vancomycin for presumed late-onset sepsis in the Neonatal Intensive Care Unit and the impact upon outcome of coagulase negative staphylococcal bacteremia: a retrospective cohort study. BMC Pediatr 2005;5:49.

28. Brandt CM, Rouse MS, Tallan BM, Laue NW et al. Effective treatment of cephalosporin-rifampin combinations against cryptic methicillin-resistant beta-lactamase-producing coagulase-negative staphylococcal experimental endocarditis. Antimicrob Agents Chemother 1995;39(8):1815-9. 